\title{
Characteristics, Risk Factors And Outcome Of Early-Onset Delirium In Elderly Patients With First Ever Acute Ischemic Stroke - A Prospective Observational Cohort Study
}

This article was published in the following Dove Press journal:

Clinical Interventions in Aging

\author{
Katarzyna Kotfis (D) \\ Marta Bott-Olejnik (iD) ${ }^{2}$ \\ Aleksandra Szylińska (D) ${ }^{3}$ \\ Mariusz Listewnik (D) ${ }^{4}$ \\ Iwona Rotter (D) ${ }^{3}$ \\ 'Department of Anesthesiology, Intensive \\ Therapy and Acute Intoxications, \\ Pomeranian Medical University, Szczecin \\ 70-204, Poland; ${ }^{2}$ Neurology Department \\ of a Regional Specialist Hospital in \\ Gryfice, Gryfice 72-300, Poland; \\ ${ }^{3}$ Department of Medical Rehabilitation \\ and Clinical Physiotherapy, Pomeranian \\ Medical University, Szczecin 70-204, \\ Poland; ${ }^{4}$ Department of Cardiac Surgery, \\ Pomeranian Medical University, Szczecin \\ 70-204, Poland
}

Introduction: Post-stroke delirium is a common clinical problem, occurring in $10 \%$ to $48 \%$ of patients. It has been associated with longer hospitalization times, increased mortality and worse functional outcome. In early phase of stroke, it may be regarded as particularly difficult to differentiate from other neurological symptoms. For practical purposes, there is a need to identify simple pre-operative laboratory parameters that may aid delirium diagnosis early after stroke. Our aim was to identify the incidence of early-onset (first $24 \mathrm{hrs)} \mathrm{post-stroke} \mathrm{delirium,} \mathrm{its} \mathrm{risk} \mathrm{factors}$ and outcomes (complications and mortality) in patients with first-ever acute ischemic stroke (AIS). Material and methods: A retrospective analysis of a prospective observational study (NCT03944694) was performed. Patients were screened for delirium using CAM-ICU method. Clinical and laboratory data were collected, including baseline inflammatory parameters.

Results: Final analysis included 760 patients, 121 (15.9\%) developed delirium. Patients with delirium were older $(75.9 \pm 13.5$ years, $\mathrm{p}<0.001)$. Most common complications in the delirium group were pulmonary $(57.8 \%$ vs $21.4 \%, \mathrm{p}<0.001)$, cardiac $(38.8 \%$ vs $13.6 \%, \mathrm{p}<0.001)$ and renal $(13.2 \%$ vs $7.5 \%, \mathrm{p}=0.038)$. Neutrophil-to-lymphocyte ratio (NLR) (6.71 \pm 9.65 vs 4.55 $\pm 5.51, \mathrm{p}<0.001)$, C-reactive protein level $(32.59 \pm 65.94$ vs $15.70 \pm 38.56, \mathrm{p}<0.001)$ and troponin T level $(72.59 \pm 180.15$ vs $26.85 \pm 77.62, \mathrm{p}<0.001)$ were higher in delirious patients and platelet-to white blood cell count ratio (PWR) $(23.42 \pm 9.51$ vs $27.13 \pm 10.58, \mathrm{p}<0.001)$ was lower. Multivariable logistic regression showed that atrial fibrillation (OR 1.651, $\mathrm{p}=0.049$ ), higher Rankin score on admission (OR 1.689, p<0.001), hemianopia (OR 2.422, p=0.003) and PWR $<20.22$ (OR 2.197, p=0.002) were independently associated with delirium. Kaplan-Meier curves indicated that mortality increased for patients with delirium at 3 months $(\mathrm{p}<0.001)$ and 1 year $(\mathrm{p}<0.001)$ after AIS.

Conclusion: Atrial fibrillation, higher Rankin score, hemianopia and lower PWR were independently associated with early onset delirium in patients with first ever AIS. This confirms that deprivation of senses and early generalized inflammatory response are critical for delirium development.

Keywords: delirium, first-ever stroke, PWR, CRP, NIHSS, CAM-ICU, acute brain dysfunction

\section{Introduction}

Acute ischemic stroke (AIS) is a neurological emergency that may lead to serious immediate and long-term physical, emotional and cognitive complications. ${ }^{1-4}$ Poststroke delirium is a common clinical problem occurring in $10 \%$ to $48 \%$ of patients. ${ }^{1,4}$
Correspondence: Katarzyna Kotfis Department of Anesthesiology, Intensive Therapy and Acute Intoxications,

Pomeranian Medical University, Szczecin, al, Powstańców Wielkopolskich 72, Szczecin 70-III, Poland

Tel/fax +48 9I 466 II 44

Email katarzyna.kotfis@pum.edu.pl 
According to a meta-analysis performed by Shi et al, patients with delirium after stroke have longer hospitalization times, are 4.7 times more likely to die in the hospital and within the first year after stroke and have poorer functional outcomes with lower quality of life. ${ }^{1}$ Post-stroke delirium is an acute neuro-psychiatric syndrome that aggravates patients' and family members' distress and leads to long-term consequences, especially at the extremes of age. ${ }^{3,4}$

Delirium is characterized by an acute onset of symptoms including altered level of consciousness and a fluctuating course with changes in orientation, memory, thinking or behavior. ${ }^{3,5}$ It may be present as a complication of infection, hypoxia, metabolic derangement or an effect of various centrally acting medications, especially in elderly patients. It may also become evident as a consequence of a medical problem, such as stroke or occur after major surgery. ${ }^{6-9}$

The stroke itself is a known predisposing factor for delirium, yet studies that have investigated this association gives conflicting results. ${ }^{4}$ In order to limit the negative consequences of delirium in stroke patients, clinicians should screen for delirium to identify its early signs, recognize modifiable precipitating factors and risk factors to improve clinical outcomes. ${ }^{10-12}$ It is recommended that screening for delirium is easy and should be active, with the use of validated psychometric tools CAM, CAM-ICU (confusion assessment method for intensive care unit) or ICDSC (Intensive Care Delirium Screening Checklist). ${ }^{11,13}$ Poststroke delirium, especially in the early phase of stroke, may be regarded as particularly difficult to recognize and differentiate from other neurological symptoms; however, usefulness of CAM-ICU to diagnose delirium in post-stroke delirium has been confirmed by many studies. ${ }^{10,14,15}$ The CAM-ICU has been validated for use in non-verbal intubated patients in the ICU, but it may also be used in other nonverbal patients including those with aphasia after stroke. ${ }^{14}$

The pathophysiology of delirium is multifactorial and not entirely understood. Mechanisms involving neuroinflammation and oxidative stress, as well as the interplay between inflammatory cytokines and the cholinergic system have been reported. ${ }^{16,17} \mathrm{~A}$ number of biomarkers has been identified in different delirium populations, yet they are used mainly in the research setting. ${ }^{8,18-20}$ There is a need for a practicing clinician to identify and use simple pre-operative laboratory parameters that may aid delirium diagnosis early after stroke. Moreover, the assessment time-point is also very important, as different values were reported in literature and the number of studies regarding early-onset delirium performed in patients with ischemic stroke is limited. ${ }^{1,4}$ A recently published prospective study performed by $\mathrm{Qu}$ et al in the first-ever ischemic brain stroke showed that delirium frequency was rather low with the incidence of $14.6 \% .^{21}$ According to Pasinska et al most cases of delirium are recognizable at the beginning of hospitalization, with $71 \%$ of patients developing post-stroke delirium within $24 \mathrm{hrs}$ after admission. $^{22,23}$

No previous study has specifically concentrated on the diagnosis of early-onset post-stroke delirium. Moreover, data regarding complications in post-stroke delirium is limited. The authors identified a need to perform a study regarding early-onset delirium in a very homogenous group of patients including only acute ischemic stroke without a previous stroke. Therefore, our aim was to identify the incidence of early onset (within $24 \mathrm{hrs}$ of admission) post-stroke delirium, its risk factors and outcome, including complications (pulmonary, cardiac, renal, other) and mortality (at 7 days, 30 days, 90 days and 1 year) in a homogenous group of patients with first-ever acute ischemic stroke.

\section{Materials And Methods Study Population And Data Collection}

We performed a secondary retrospective analysis of a prospective observational cohort study (DELirium In Acute ischemic Stroke, DELIAS Study, NCT03944694). Patients of both sexes, aged $>18$ years, with acute ischemic stroke who were admitted to the stroke unit of a district general hospital within 48 hrs after developing neurological symptoms, were screened for inclusion into the study. Neurological deficits were assessed using the National Institutes of Health Stroke Scale (NIHSS) at admission and discharge. Neuroimaging was performed using brain computed tomography (CT) on admission, with lesions classified into one of the four categories: no lesion, lesion $<2.5 \mathrm{~cm}$ (supratentorial and infratentorial), lesion $>2.5 \mathrm{~cm}$, lacunar lesion (supratentorial lesions). Functional ability was assessed with the use of modified Rankin Scale (mRS) at admission and discharge. Data were collected prospectively by a member of the study team and included: complete past medical history, comorbidities, clinical and neurological evaluation, carotid artery ultrasonography, laboratory results (full blood count with differential white blood cell count, C-reactive protein, Troponin $\mathrm{T}$ level, serum creatinine). The leucocyte count 
(white blood cell count, WBC), neutrophil, lymphocyte and platelet counts were determined on a standard hematology analyzer with flow cytometry (Sysmex XN 2000, 2014, Japan). The neutrophil-to-lymphocyte ratio (NLR), platelet-to-neutrophil ratio (PNR) and platelet-to-WBC ratio (PWR) were derived directly from the differential WBC. The NLR was calculated by dividing the absolute neutrophil count by the absolute lymphocyte count. The PNR was calculated by dividing the platelet count over the neutrophil count and the PWR was calculated by dividing the platelet count by the WBC.

The follow-up included data extracted from medical records during patient hospitalization. Complications were recorded and categorized as: 1. pulmonary (pneumonia, respiratory failure, pulmonary embolism, bronchospasm), 2. cardiac (heart failure, myocardial infarction, sudden cardiac arrest, atrial fibrillation), 3. renal (acute renal failure, urinary tract infection), 4. neurological (seizures, CNS hemorrhage) 5. deep venous thrombosis (DVT), 6. gastrointestinal bleeding, 7. bed sores and 8. sepsis. Mortality was evaluated at 7, 30, 90 days and at 1 year after discharge, using a telephone interview. The primary endpoint was delirium diagnosed within $24 \mathrm{hrs}$ from admission in patients with first onset acute ischemic stroke.

A total of 1022 patients with AIS admitted to the hospital were screened in this study. We excluded 241 patients with a history of previous stroke, 5 patients with hematology disorders, 6 patients with incomplete laboratory results and 10 patients with no data regarding follow-up (Figure 1); therefore, 760 patients with firstever stroke were included in the final analysis.

\section{Delirium Screening}

All patients were screened for delirium on admission and during their hospitalization twice daily (9:00 and 19:00) after admission to the neurology (stroke) unit. The cognitive assessment method for ICU (CAM-ICU) was used because it allows assessment of all, even nonverbal patients. We used the Polish language version of the CAM-ICU test. ${ }^{13}$ To facilitate the diagnosis of delirium, medical and nursing notes were reviewed by one of the investigators. The diagnosis of delirium was made using the Diagnostic and Statistical Manual of Mental Disorders 5th edition. ${ }^{5}$ We used "early onset" definition, if delirium was diagnosed within the first $24 \mathrm{hrs}$ after admission with AIS to the neurology unit.

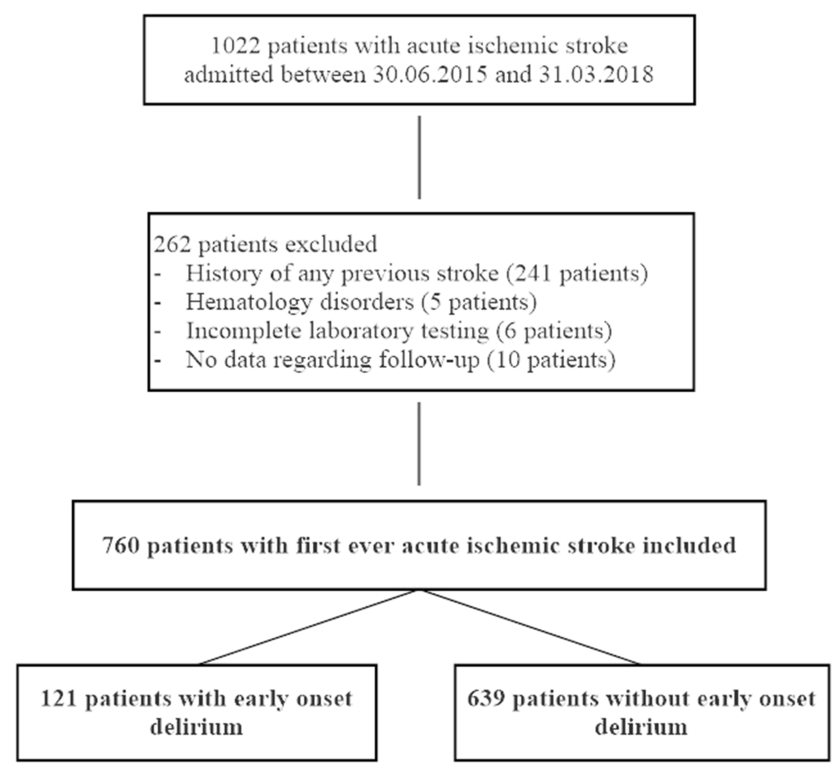

Figure I Study flow-chart.

\section{Ethical Issues}

The study was performed according to the Declaration of Helsinki and an approval of the Bioethical Committee of the Pomeranian Medical University (KBE-0012/84/03/19) was granted. The data were coded and analyzed anonymously. The study was registered at Clinical Trials Gov register (NCT03944694).

\section{Statistical Analysis}

The data are presented as numbers and percentages for categorical variables and as means and standard deviations for continuous variables. The cohort of first-ever acute ischemic stroke patients was divided according to the absence or presence of early-onset delirium on admission (Delirium vs No Delirium group). Chi-square test was used to compare qualitative data between patients with and without delirium. Mann Whitney $U$-test was used to compare continuous variables. The receiver-operating characteristics curve (ROC) analysis was performed to determine the best cut-off value for laboratory parameters predicting the clinical endpoints. Univariable and multivariable logistic regression analysis was performed with delirium occurrence as the dependent variable to analyze independent risk factors and calculate the odds ratios (OR). Kaplan-Meier analysis calculated the probability of survival. The p-value was considered significant at the level $\leq 0.05$. All statistical analyses were performed with the use of a licensed software Statistica 13 (StatSoft, Inc. Tulsa, OK, USA). 


\section{Results}

The final analysis included 760 patients, 121 (15.92\%) developed delirium within $24 \mathrm{hrs}$ of the onset of acute ischemic stroke. Patients with delirium were older $(75.95 \pm 13.49$ years vs $70.82 \pm 12.15$ years, $\mathrm{p}<0.001)$, there were no differences between sexes in the study sample. More patients with delirium suffered from ischemic heart disease $(32.23 \%$ vs $22.38 \%, \mathrm{p}=0.019)$, congestive heart failure $(42.50 \%$ vs $24.41 \%, \mathrm{p}<0.001)$, transient ischemic attack prior to current stroke $(11.57 \%$ vs $5.95 \%, \mathrm{p}=0.025)$, had acute renal failure on admission $(3.31 \%$ vs $0.31 \%, p=0.004)$, impaired glucose tolerance $(9.09 \%$ vs $3.44 \%, \mathrm{p}=0.005)$, extracardiac arteriopathy $(57.02 \%$ vs $37.09 \%, \mathrm{p}<0.001)$ and atrial fibrillation $(48.76 \%$ vs $25.08 \%, \mathrm{p}<0.001)$ as depicted in Table 1.

Patients with delirium used ADP inhibitors (5.79\% vs $1.56 \%, \mathrm{p}=0.011)$ and diuretics $(44.63 \%$ vs $34.27 \%$, $\mathrm{p}=0.029)$ significantly more often than patients without delirium (Table 2). On the other hand, ACE-inhibitors or sartans $(40.50 \%$ vs $55.71 \%, \mathrm{p}=0.002)$, as well as calcium channel blockers $(16.53 \%$ vs $27.23 \%, \mathrm{p}=0.013)$ were used less often in patients with delirium.
Baseline neurology data is shown in Table 3. Patients with delirium had higher Rankin score $(4.25 \pm 1.21$ vs $2.74 \pm$ $1.56, \mathrm{p}<0.001)$ and NIHSS score $(16.40 \pm 7.19$ vs $9.67 \pm$ $7.46, \mathrm{p}<0.001)$ on admission. Delirious patients more often presented with hemianopia $(71.07 \%$ vs $29.11 \%$, p $<0.001)$ and dysphasia $(61.16 \%$ vs $48.98 \%, \mathrm{p}<0.001)$. There were no differences in the occurrence of brainstem stroke or secondary hemorrhage between the two groups. The CT scan showed that the majority of delirious patients presented with lesions larger than $2.5 \mathrm{~cm}(77.69 \%$ vs $42.88 \%$, p < $0.001)$, with predominance of stroke from middle cerebral artery area $(80.00 \%$ vs $61.17 \%, \mathrm{p}<0.001)$. There were no differences regarding the type of stroke treatment between both groups, majority of patients were treated conservatively.

Among patients with delirium, the most prevalent type was hyperactive form $(48.76 \%)$, followed by hypoactive type $(33.05 \%)$; only $18 \%$ of patients were diagnosed with mixed delirium (Table 4). In one third of patients, delirium persisted until discharge (29.75\%). Majority of delirious patients received non-pharmacological treatment $(36.36 \%)$, with only $8.26 \%$ receiving medications.

Table I Baseline Demographic Data And Co-Morbidities Of Patients With And Without Delirium

\begin{tabular}{|c|c|c|c|}
\hline Variables & Delirium $(n=121)$ & No Delirium $(n=639)$ & p-value \\
\hline \multicolumn{4}{|l|}{ Demographic data } \\
\hline Age [years], mean $\pm S D$ & $75.95 \pm 13.49$ & $70.82 \pm 12.15$ & $<0.001$ \\
\hline Gender [male], n (\%) & $56(46.28)$ & $337(52.74)$ & 0.192 \\
\hline BMI $\left[\mathrm{kg} / \mathrm{m}^{2}\right]$, mean $\pm \mathrm{SD}$ & $27.06 \pm 4.76$ & $26.67(4.79)$ & 0.196 \\
\hline Smoking, n (\%) & $53(43.80)$ & $266(41.63)$ & 0.657 \\
\hline \multicolumn{4}{|l|}{ Co-morbidities } \\
\hline Arterial hypertension, $\mathrm{n}(\%)$ & $100(82.64)$ & $543(84.98)$ & 0.515 \\
\hline Ischemic heart diseases, $\mathrm{n}(\%)$ & $39(32.23)$ & $143(22.38)$ & 0.019 \\
\hline Myocardial infarction, $\mathrm{n}(\%)$ & II (9.09) & $65(10.17)$ & 0.716 \\
\hline Congestive heart failure, $\mathrm{n}(\%)$ & $51(42.50)$ & $156(24.41)$ & $<0.001$ \\
\hline TIA during last 30 days, $\mathrm{n}(\%)$ & $16(13.22)$ & $63(9.86)$ & 0.266 \\
\hline TIA earlier than last 30 days, $n$ (\%) & $14(11.57)$ & $38(5.95)$ & 0.025 \\
\hline Acute Renal Failure on admission, $\mathrm{n}$ (\%) & $4(3.31)$ & $2(0.31)$ & 0.004 \\
\hline Chronic Renal Failure, n (\%) & $21(17.36)$ & $85(13.30)$ & 0.299 \\
\hline Chronic Dialysis, $\mathrm{n}(\%)$ & $0(0.00)$ & $2(0.31)$ & 0.725 \\
\hline Impaired insulin tolerance, $\mathrm{n}(\%)$ & II (9.09) & $22(3.44)$ & 0.005 \\
\hline Diabetes on oral medications, $\mathrm{n}$ (\%) & $25(20.66)$ & I2I (I8.94) & 0.659 \\
\hline Diabetes on insulin, $\mathrm{n}(\%)$ & $18(14.88)$ & $80(12.54)$ & 0.482 \\
\hline Chronic obstructive pulmonary disease & $12(9.92)$ & $54(8.45)$ & 0.727 \\
\hline Extracardiac arteriopathy, $\mathrm{n}(\%)$ & $69(57.02)$ & $237(37.09)$ & $<0.001$ \\
\hline Atrial Fibrillation, $\mathrm{n}(\%)$ & $59(48.76)$ & $260(25.08)$ & $<0.001$ \\
\hline AF paroxysmal, n (\%) & $7(5.79)$ & $36(5.63)$ & 0.882 \\
\hline $\mathrm{AF}$ persistent, $\mathrm{n}(\%)$ & $42(34.7 I)$ & $87(13.62)$ & $<0.001$ \\
\hline
\end{tabular}

Abbreviations: $n$, number of patients; SD, standard deviation; BMI, body mass index; TIA, transient ischemic attack; AF, atrial fibrillation. 
Table 2 Medications Used Prior To Stroke In Patients With And Without Delirium

\begin{tabular}{|c|c|c|c|}
\hline Medications & Delirium $(n=121)$ & No Delirium $(n=639)$ & p-value \\
\hline Aspirine, n (\%) & 71 (58.68) & $332(51.96)$ & 0.208 \\
\hline ADP Inhibitors, $n$ (\%) & $7(5.79)$ & $10(1.56)$ & 0.011 \\
\hline Vitamin $\mathrm{K}$ antagonists, $\mathrm{n}(\%)$ & II (9.09) & $36(5.63)$ & 0.214 \\
\hline NOAC, n (\%) & $3(2.48)$ & $7(1.10)$ & 0.429 \\
\hline Beta-blockers, n (\%) & $59(48.76)$ & $28 I(43.97)$ & 0.332 \\
\hline ACE-I/Sartans, n (\%) & $49(40.50)$ & $356(55.7 I)$ & 0.002 \\
\hline Calcium channel blockers, n (\%) & $20(16.53)$ & $174(27.23)$ & 0.013 \\
\hline Statins, n (\%) & $26(21.49)$ & $166(25.98)$ & 0.353 \\
\hline Nitrates, n (\%) & $3(2.48)$ & $10(1.56)$ & 0.742 \\
\hline Diuretics, n (\%) & $54(44.63)$ & $219(34.27)$ & 0.029 \\
\hline Aldosterone receptor antagonists, $\mathrm{n}$ (\%) & $8(6.6 I)$ & $28(4.38)$ & 0.409 \\
\hline Endothelin inhibitor/PDE, n (\%) & $0(0.00)$ & $2(0.3 I)$ & 0.725 \\
\hline Bronchodilators, n (\%) & I $(0.83)$ & $22(3.44)$ & 0.211 \\
\hline Oral diabetic medications, n (\%) & $20(16.53)$ & $110(17.21)$ & 0.959 \\
\hline Insuline, n (\%) & $16(13.22)$ & $68(10.64)$ & 0.501 \\
\hline
\end{tabular}

Abbreviations: $\mathrm{n}$, number of patients; NOAK, new oral anticoagulants.

Table 3 Baseline Neurology Data For AIS Patients With And Without Delirium

\begin{tabular}{|c|c|c|c|c|}
\hline \multicolumn{2}{|l|}{ Variables } & Delirium $(n=121)$ & No Delirium $(n=639)$ & p-value \\
\hline \multicolumn{2}{|c|}{ Rankin score at admission, mean $\pm S D$} & $4.25 \pm 1.21$ & $2.74 \pm 1.56$ & $<0.001$ \\
\hline \multicolumn{2}{|l|}{ NIHSS on admission, mean \pm SD } & $16.40 \pm 7.19$ & $9.67 \pm 7.46$ & $<0.001$ \\
\hline \multicolumn{2}{|l|}{ Hemianopia, n (\%) } & $86(71.07)$ & $186(29.11)$ & $<0.001$ \\
\hline \multicolumn{2}{|l|}{ Dysphasia, n (\%) } & $74(61.16)$ & $313(48.98)$ & 0.014 \\
\hline \multicolumn{2}{|l|}{ Brainstem stroke, n (\%) } & $5(4.13)$ & $34(5.32)$ & 0.749 \\
\hline \multicolumn{2}{|l|}{ Secondary hemorrhage, n (\%) } & $6(4.96)$ & $19(2.97)$ & 0.398 \\
\hline Paresis, n (\%) & $\begin{array}{l}\text { Right-sided } \\
\text { Left-sided } \\
\text { Bilateral }\end{array}$ & $\begin{array}{l}59(51.75) \\
52(45.61) \\
3(2.63)\end{array}$ & $\begin{array}{l}292(51.05) \\
266(46.50) \\
14(2.45)\end{array}$ & 0.981 \\
\hline CT scan, n (\%) & $\begin{array}{l}\text { No lesion } \\
\text { Lesion up to } 2.5 \mathrm{~cm} \\
\text { Lesion above } 2.5 \mathrm{~cm} \\
\text { Lacunar stroke }\end{array}$ & $\begin{array}{l}7(5.79) \\
20(16.53) \\
94(77.69) \\
0(0.00)\end{array}$ & $\begin{array}{l}50(7.82) \\
297(46.48) \\
274(42.88) \\
18(2.82)\end{array}$ & $<0.001$ \\
\hline Cerebral artery area, $\mathrm{n}(\%)$ & $\begin{array}{l}\text { Anterior } \\
\text { Middle } \\
\text { Posterior }\end{array}$ & $\begin{array}{l}6(5.00) \\
96(80.00) \\
18(15.00)\end{array}$ & $\begin{array}{l}143(22.66) \\
386(61.17) \\
102(16.16)\end{array}$ & $<0.001$ \\
\hline Carotid artery stenosis, n (\%) & $\begin{array}{l}\text { No stenosis } \\
\text { Stenosis up to } 50 \% \\
\text { Stenosis } 50-70 \% \\
\text { Stenosis }>70 \% \\
\text { Occlusion }\end{array}$ & $\begin{array}{l}3(2.63) \\
56(49.12) \\
43(37.72) \\
4(3.51) \\
8(7.02)\end{array}$ & $\begin{array}{l}24(3.90) \\
391(63.58) \\
135(21.95) \\
36(5.85) \\
29(4.72)\end{array}$ & 0.004 \\
\hline Stroke treatment, n (\%) & $\begin{array}{l}\text { Conservative } \\
\text { Thrombolysis }\end{array}$ & $\begin{array}{l}103(85.83) \\
17(14.17)\end{array}$ & $\begin{array}{l}478(78.36) \\
132(21.64)\end{array}$ & 0.063 \\
\hline
\end{tabular}

Abbreviations: NIHSS, National Institute of Health Stroke Scale; $n$, number of patients; SD, standard deviation; CT, computed tomography. 
Table 4 Data Regarding Delirium Types And Treatment

\begin{tabular}{|l|l|l|}
\hline Variables & & n (\%) \\
\hline Delirium type & Hyperactive & $59(48.76)$ \\
& Hypoactive & $40(33.05)$ \\
& Mixed & $22(18.18)$ \\
\hline Persistent delirium (until discharge) & $36(29.75)$ \\
\hline Delirium treatment & Non-pharmacological & $44(36.36)$ \\
& Pharmacological & $10(8.26)$ \\
& No treatment & $67(55.37)$ \\
\hline Medications used & Haloperidol & $1(0.83)$ \\
& Quetiapine & $6(4.96)$ \\
& Other & $3(2.48)$ \\
\hline
\end{tabular}

Abbreviation: $n$, number of patients.

The results of standard laboratory tests performed on admission to the hospital are shown in Table 5. In patients with delirium, the total leucocyte count $(10.77 \pm 5.56 \mathrm{vs}$ $9.55 \pm 3.65, \mathrm{p}=0.018)$ and neutrophil count $(7.89 \pm 4.76$ vs $6.65 \pm 3.47, \mathrm{p}=0.001)$ was higher, but the lymphocyte count $(1.91 \pm 2.04$ vs $2.01 \pm 1.15, \mathrm{p}=0.003)$ and platelet count $(223.66 \pm 75.74$ vs $241.59 \pm 92.94, \mathrm{p}=0.018)$ was lower in patients with delirium. The derived parameters also showed significant differences - the NLR was higher $(6.71 \pm 9.65$ vs $4.55 \pm 5.51, \mathrm{p}<0.001)$ and PWR was lower $(23.42 \pm 9.51$ vs $27.13 \pm 10.58, \mathrm{p}<0.001)$ in patients with delirium. The C-reactive protein level was elevated in both groups, yet significantly higher in delirious patients $(32.59 \pm 65.94$ vs $15.70 \pm 38.56$, p $<0.001)$. Moreover, the troponin $\mathrm{T}$ level was elevated in delirium $(72.59 \pm 180.15$ vs $26.85 \pm 77.62, \mathrm{p}<0.001)$.
We used ROC analysis to find the cut-off values for derived inflammatory ratios and parameters of interest and found that the cut-off for diagnosing delirium within the first 24 hrs after stroke are: NLR $>4.86$, PWR $<20.22$ and CRP $>6.10$ (Figure 2).

Outcome data for patients with and without delirium are shown in Table 6. Hospitalization time for patients with delirium was longer (12.12 \pm 7.50 days vs $10.32 \pm$ 6.76 days, $\mathrm{p}<0.001)$ and were discharged with worse scores as identified by NIHSS $(17.27 \pm 14.25$ vs $8.87 \pm$ $12.14, \mathrm{p}<0.001)$ and Rankin scale $(3.95 \pm 1.85$ vs $2.20 \pm$ $2.22, \mathrm{p}<0.001)$. The mortality was higher for delirious patients as reported at day $7(13.22 \%$ vs $5.63 \%$, p $=$ $0.005)$, at day $30(33.88 \%$ vs $12.83 \%, \mathrm{p}<0.001)$ and year one of observation $(57.02 \%$ vs $23.63 \%$, p $<0.001)$. In the delirium group $24 / 121$ patients $(19.83 \%)$ died during hospitalization as opposed to $56 / 639(8.76 \%)$ of nondelirious patients $(\mathrm{p}<0.001)$. Patients with delirium were less likely to be discharged home that patients without delirium $(39.67 \%$ vs $62.60 \%, \mathrm{p}<0.001)$.

We performed a detailed analysis of complications that occurred after stroke and during hospitalization (Table 7). More than twice as many patients with delirium developed any kind of complications ( $76.03 \%$ vs $30.36 \%$, p < 0.001$)$. In patients with delirium the most common complications were of pulmonary origin $(57.85 \%$ vs $21.44 \%, \mathrm{p}<0.001)$, followed by cardiac $(38.84 \%$ vs $13.62 \%, \mathrm{p}<0.001)$ and renal problems $(13.22 \%$ vs $7.51 \%, \mathrm{p}=0.038)$.

In order to identify risk factors associated with early onset delirium in patients with first ever acute ischemic stroke a univariable and multivariable logistic regression

Table 5 Laboratory Results On Admission In Patients With And Without Delirium

\begin{tabular}{|l|l|l|l|}
\hline Laboratory Results On Admission & Delirium, Mean \pm SD $(\mathbf{n}=\mathbf{~ I 2 I )}$ & No delirium, Mean \pm SD $(\mathbf{n}=\mathbf{6 3 9})$ & $\mathbf{p}$-value \\
\hline Leucocyte count $\left(\times 10^{9} / \mathrm{L}\right)$ & $10.77 \pm 5.56$ & $9.55 \pm 3.65$ & 0.018 \\
Neutrophil count $\left(\times 10^{9} / \mathrm{L}\right)$ & $7.89 \pm 4.76$ & $6.65 \pm 3.47$ & $0.00 \mathrm{I}$ \\
Lymphocyte count $\left(\times 10^{9} / \mathrm{L}\right)$ & $1.91 \pm 2.04$ & $2.01 \pm 1.15$ & 0.003 \\
Platelet count $\left(\times 10^{9} / \mathrm{L}\right)$ & $223.66 \pm 75.74$ & $241.59 \pm 92.94$ & 0.018 \\
Neutrophil-to-lymphocyte ratio & $6.71 \pm 9.65$ & $4.55 \pm 5.51$ & $<0.00 \mathrm{I}$ \\
Platelet-to-neutrophil ratio & $35.16 \pm 19.46$ & $42.38 \pm 20.85$ & $<0.00 \mathrm{I}$ \\
Platelet-to-WBC ratio & $23.42 \pm 9.5 \mathrm{I}$ & $27.13 \pm 10.58$ & $<0.00 \mathrm{I}$ \\
Hemoglobin & $13.87 \pm 1.88$ & $13.88 \pm 1.85$ & 0.610 \\
Creatinine & $1.14 \pm 0.59$ & $1.06 \pm 0.66$ & 0.022 \\
C-reactive protein & $32.59 \pm 65.94$ & $15.70 \pm 38.56$ & $<0.00 \mathrm{I}$ \\
Aspartate aminotransferase & $49.91 \pm 139.17$ & $24.21 \pm 14.83$ & $0.00 \mathrm{I}$ \\
Alanine aminotransferase & $38.56 \pm 92.60$ & $22.17 \pm 14.75$ & 0.100 \\
Troponin T & $72.59 \pm 180.15$ & $26.85 \pm 77.62$ & $<0.00 \mathrm{I}$ \\
\hline
\end{tabular}

Abbreviations: $n$, number of patients; SD, standard deviation; NLR, neutrophil-to-lymphocyte ratio, CRP, C-reactive protein. 


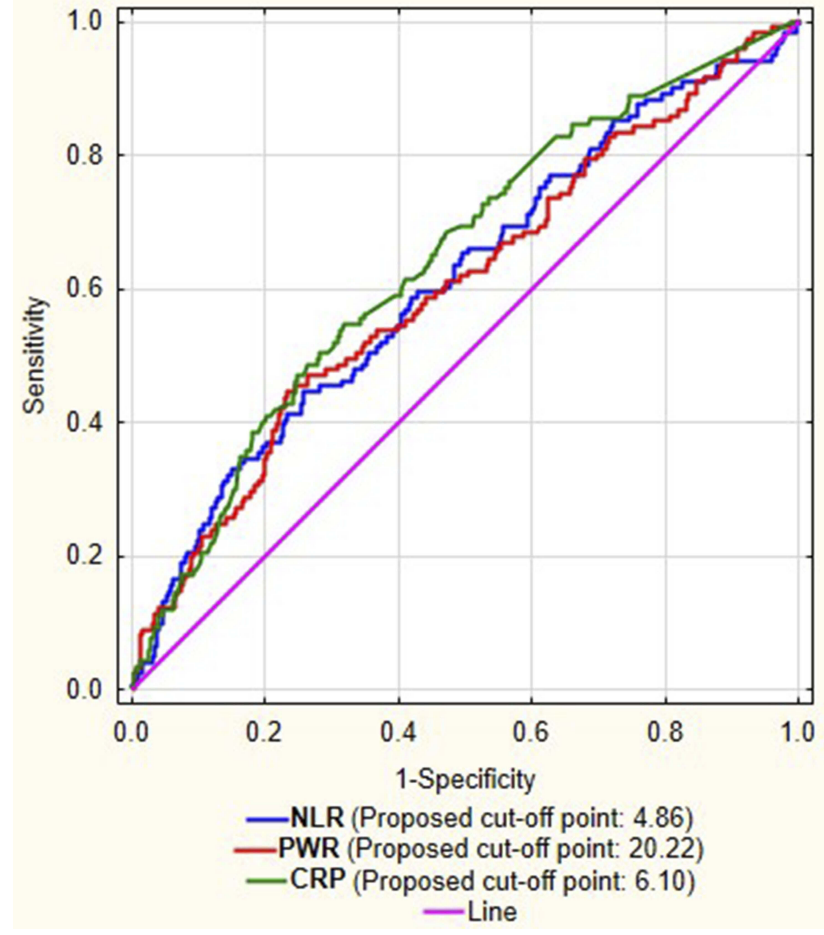

Figure 2 Receiver-operator curves for NLR, PWR and CRP with their cut-off points.

analysis was performed (Table 8 ). We used variables with highest statistical significance $(\mathrm{p}<0.001)$ in the univariable analysis for multivariable analysis. Only a history of atrial fibrillation (OR 1.651, 95\% CI 1.003-2.719, $\mathrm{p}=0.049$ ), higher Rankin score on admission (OR 1.689, 95\% CI $1.280-2.229, \mathrm{p}<0.001)$, hemianopia as a presenting syndrome (OR 2.422, 95\% CI 1.351-4.342, $\mathrm{p}=0.003$ ) and
platelet-to-WBC ratio below 20.22 (OR 2.197, 95\% CI 1.347-3.601, p $=0.002$ ) were independently associated with early onset delirium in patients with first ever acute ischemic stroke in the multivariable analysis.

The Kaplan-Meier curves visible in Figure 3 show that the probability of survival until day 90 . after stroke was significantly lower in patients with delirium $(\mathrm{p}<0.001)$. Similarly, the Kaplan-Meier curves regarding the probability of survival until 1 year after stroke showed significantly ( $p<0.001)$ worse result for patients with delirium as shown in Figure 4.

\section{Discussion}

Post-stroke delirium is a serious clinical problem with long-term consequences. The number of studies regarding delirium in patients with first-ever ischemic stroke is very limited. $^{24-26}$ The results of this retrospective analysis of a prospective cohort study (DELIAS Study) including 760 patients revealed that the incidence of delirium diagnosed within the first day of admission to the hospital was nearly $16 \%$, with half $(48.7 \%)$ of patients presenting with hyperactive delirium. Complications during hospital stay occurred more frequently in patients with delirium than in patients without delirium $(76 \%$ vs $30 \%, \mathrm{p}<0.001)$, the most common being pulmonary $(58 \%)$, cardiac $(39 \%)$ or renal $(13 \%)$ in origin.

The hospitalization time for patients with delirium was significantly longer. They were discharged from the neurology unit with worse NIHSS score and with greater disability as

Table 6 Outcome Data For Patients With And Without Delirium

\begin{tabular}{|c|c|c|c|c|}
\hline \multicolumn{2}{|l|}{ Variables } & Delirium (n = |2|) & No Delirium $(n=639)$ & p-value \\
\hline \multicolumn{2}{|c|}{ Hospitalization time (days), mean $\pm S D$} & $12.12 \pm 7.50$ & $10.32 \pm 6.76$ & $<0.001$ \\
\hline \multicolumn{2}{|c|}{ NIHSS at discharge, mean \pm SD } & $17.27 \pm 14.25$ & $8.87 \pm 12.14$ & $<0.001$ \\
\hline \multicolumn{2}{|c|}{ Rankin score at discharge, mean $\pm S D$} & $3.95 \pm 1.85$ & $2.20 \pm 2.22$ & $<0.001$ \\
\hline \multicolumn{2}{|c|}{ Mortality until day $7, \mathrm{n}(\%)$} & $16(13.22)$ & $36(5.63)$ & 0.005 \\
\hline \multicolumn{2}{|c|}{ Mortality until day $30, \mathrm{n}(\%)$} & $41(33.88)$ & $82(12.83)$ & $<0.001$ \\
\hline \multicolumn{2}{|c|}{ Mortality until day $90, \mathrm{n}(\%)$} & $57(47.11)$ & $118(18.47)$ & $<0.001$ \\
\hline \multicolumn{2}{|c|}{ Mortality until year I, n (\%) } & $69(57.02)$ & $151(23.63)$ & $<0.001$ \\
\hline Outcome & $\begin{array}{l}\text { In-hospital death, n (\%) } \\
\text { Discharged home, n (\%) } \\
\text { Nursing home, n (\%) } \\
\text { Rehabilitation facility, n (\%) } \\
\text { Other ward, n (\%) }\end{array}$ & $\begin{array}{l}24(19.83) \\
48(39.67) \\
19(15.70) \\
27(22.31) \\
3(2.48)\end{array}$ & $\begin{array}{l}56(8.76) \\
400(62.60) \\
42(6.57) \\
131(20.50) \\
10(1.56)\end{array}$ & $<0.001$ \\
\hline
\end{tabular}

Abbreviations: $n$, number of patients; SD, standard deviation; NIHSS, National Institutes of Health Stroke Scale. 
Table 7 Post-Stroke Complications In Delirious And Non-Delirious Patients

\begin{tabular}{|c|c|c|c|c|}
\hline \multicolumn{2}{|l|}{ Complications } & Delirium, $n(\%)(n=12 \mid)$ & No Delirium, $n(\%)(n=639)$ & p-value \\
\hline \multicolumn{2}{|l|}{ Any complications } & $92(76.03)$ & $194(30.36)$ & $<0.001$ \\
\hline \multicolumn{2}{|c|}{ Cardiological complications } & $47(38.84)$ & $87(13.62)$ & $<0.001$ \\
\hline \multicolumn{2}{|l|}{ Heart failure } & $38(31.40)$ & $61(9.55)$ & $<0.001$ \\
\hline \multicolumn{2}{|l|}{ Myocardial infarction } & $9(7.44)$ & $16(2.50)$ & 0.012 \\
\hline \multicolumn{2}{|l|}{ NSTEMI } & $9(7.44)$ & $15(2.35)$ & 0.008 \\
\hline \multicolumn{2}{|l|}{ STEMI } & $0(0.00)$ & I (0.16) & $0.35 \mathrm{I}$ \\
\hline \multicolumn{2}{|c|}{ Sudden cardiac arrest } & $25(20.66)$ & $50(7.82)$ & $<0.001$ \\
\hline \multicolumn{2}{|l|}{ Atrial fibrillation } & $3(2.48)$ & $10(1.56)$ & 0.742 \\
\hline \multicolumn{2}{|c|}{ Pulmonary complications } & $70(57.85)$ & $137(2 \mid .44)$ & $<0.001$ \\
\hline \multicolumn{2}{|l|}{ Respiratory failure } & $27(22.31)$ & $48(7.5 \mathrm{I})$ & $<0.001$ \\
\hline \multicolumn{2}{|l|}{ Pneumonia } & $59(48.76)$ & $98(15.34)$ & $<0.001$ \\
\hline \multicolumn{2}{|l|}{ Pulmonary embolism } & $\mathrm{I}(0.83)$ & $2(0.31)$ & 0.972 \\
\hline \multicolumn{2}{|l|}{ Bronchospasm } & $4(3.31)$ & $20(3.13)$ & 0.856 \\
\hline \multicolumn{2}{|c|}{ Renal complications } & $16(13.22)$ & $48(7.5 \mathrm{I})$ & 0.038 \\
\hline \multicolumn{2}{|l|}{ ARF } & $5(4.13)$ & $3(0.47)$ & 0.002 \\
\hline \multicolumn{2}{|c|}{ Exacerbation of chronic renal failure } & $2(1.65)$ & II (I.72) & 0.742 \\
\hline \multicolumn{2}{|c|}{ Hemofiltration in ARF } & $\mathrm{I}(0.83)$ & $0(0.00)$ & 0.351 \\
\hline \multicolumn{2}{|c|}{ Conservative treatment in ARF } & $4(3.31)$ & $3(0.47)$ & 0.013 \\
\hline \multicolumn{2}{|l|}{ UTI } & $13(10.74)$ & $34(5.32)$ & 0.023 \\
\hline \multicolumn{2}{|c|}{ Neurological complications } & II (9.09) & $23(3.60)$ & 0.007 \\
\hline \multicolumn{2}{|l|}{ Seizures } & $4(3.31)$ & $8(1.26)$ & 0.210 \\
\hline CNS Hemorrhage & $\begin{array}{l}\text { No hemorrhage } \\
\text { In conservative treatment group } \\
\text { In thrombolytic treatment group }\end{array}$ & $\begin{array}{l}119(98.35) \\
0(0.00) \\
2(1.65)\end{array}$ & $\begin{array}{l}629(98.59) \\
5(0.78) \\
4(0.63)\end{array}$ & 0.316 \\
\hline \multicolumn{2}{|c|}{ Other complications } & $12(9.92)$ & $22(3.44)$ & 0.004 \\
\hline \multicolumn{2}{|l|}{ Bed sores } & $10(8.26)$ & II (I.72) & $<0.001$ \\
\hline DVT & $\begin{array}{l}\text { No DVT } \\
\text { In conservative treatment group } \\
\text { In thrombolytic treatment group }\end{array}$ & $\begin{array}{l}\text { I20 (99.17) } \\
\text { I }(0.83) \\
0(0.00)\end{array}$ & $\begin{array}{l}635(99.37) \\
3(0.47) \\
I(0.16)\end{array}$ & 0.804 \\
\hline GI bleeding & $\begin{array}{l}\text { No Gl bleeding } \\
\text { In conservative treatment group } \\
\text { In thrombolytic treatment group }\end{array}$ & $\begin{array}{l}\text { II } 17(96.69) \\
3(2.48) \\
\text { I }(0.83)\end{array}$ & $\begin{array}{l}630(98.59) \\
8(1.25) \\
I(0.16)\end{array}$ & 0.243 \\
\hline Sepsis & & $4(3.33)$ & $6(0.94)$ & 0.094 \\
\hline
\end{tabular}

Abbreviations: $\mathrm{n}$, number of patients; CNS, central nervous system; $\mathrm{Gl}$, gastro-intestinal; $\mathrm{ARF}$, acute renal failure; UTI, urinary tract infection; DVT, deep venous thrombosis.

defined by Rankin scale. The mortality in delirious patients almost tripled at day $30(34 \%$ vs $13 \%, \mathrm{p}<0.001)$ and more than doubled at one year of observation $(57 \%$ vs $24 \%$, $\mathrm{p}<0.001)$. In the delirium group twice as many patients $(20 \%)$ died during hospitalization as opposed to $(9 \%)$ nondelirious patients $(\mathrm{p}<0.001)$. Patients with delirium were less 
Table 8 Univariable And Multivariable Logistic Regression Analysis For Patients With Delirium

\begin{tabular}{|c|c|c|c|c|}
\hline Variables & Univariable Analysis OR (Cl $\pm 95 \%)$ & p-value & Multivariable Analysis OR ( $\mathrm{Cl} \pm 95 \%)$ & p-value \\
\hline Age & $1.036(1.019-1.054)$ & $<0.001$ & $1.008(0.987-1.029)$ & 0.467 \\
\hline Congestive heart failure & $2.288(1.527-3.429)$ & $<0.001$ & $1.192(0.710-2.000)$ & 0.507 \\
\hline Atrial fibrillation & $2.843(1.908-4.237)$ & $<0.001$ & $1.651(1.003-2.719)$ & 0.049 \\
\hline Extracardiac arteriopathy & $2.251(1.518-3.338)$ & $<0.001$ & $1.07 \mid(0.655-1.750)$ & 0.786 \\
\hline NIHSS on admission & $1.102(1.076-1.129)$ & $<0.001$ & $0.970(0.925-1.017)$ & 0.212 \\
\hline Rankin score on admission & $2.025(1.722-2.383)$ & $<0.001$ & $1.689(1.280-2.229)$ & $<0.001$ \\
\hline Hemianopia & $5.984(3.899-9.185)$ & $<0.001$ & $2.422(1.35 \mathrm{I}-4.342)$ & 0.003 \\
\hline NLR at cut-off $>4.86$ & $2.257(1.5 / 2-3.370)$ & $<0.001$ & $0.924(0.557-1.534)$ & 0.760 \\
\hline PWR at cut-off $<20.22$ & $2.54 \mathrm{I}(1.698-3.802)$ & $<0.001$ & $2.197(1.347-3.601)$ & 0.002 \\
\hline CRP at cut-off $>6.10$ & $2.593(1.736-3.873)$ & $<0.001$ & $2.593(0.857-2.221)$ & 0.186 \\
\hline Troponin T level & $1.003(1.001-1.005)$ & $<0.001$ & $1.001(1.000-1.003)$ & 0.141 \\
\hline
\end{tabular}

Note: Variables in the multivariable analysis were adjusted according to age, sex and BMI.

Abbreviations: Cl, confidence interval; CRP, C-reactive protein; NIHSS, National Institute of Health Stroke Scale; NLR, neutrophil-to-lymphocyte ratio; OR, odds ratio; PWR, platelet-to-WBC ratio; WBC, white blood count.

likely to be discharged home than patients without delirium. The risk factors for delirium development within $24 \mathrm{hrs}$ of admission due to first-ever acute ischemic stroke were: a history of atrial fibrillation (OR 1.651, p $=0.049$ ), higher Rankin score on admission (OR 1.689, p < 0.001), but the strongest predicting factors were hemianopia as a presenting syndrome (OR 2.422, $\mathrm{p}=0.003)$ and platelet-to-WBC ratio below 20.22 (OR 2.197, $\mathrm{p}=0.002$ ). Therefore, visual disturbance and decreased level of platelets along with elevated leucocyte count are strongly and independently associated with early onset delirium in patients with first ever acute ischemic stroke. This confirms that the deprivation of senses (hemianopia) and early generalized inflammatory response (decreased PWR) are critical for development of delirium.

Other studies regarding post-stroke delirium have shown that age, elevated admission troponin level or nicotine use

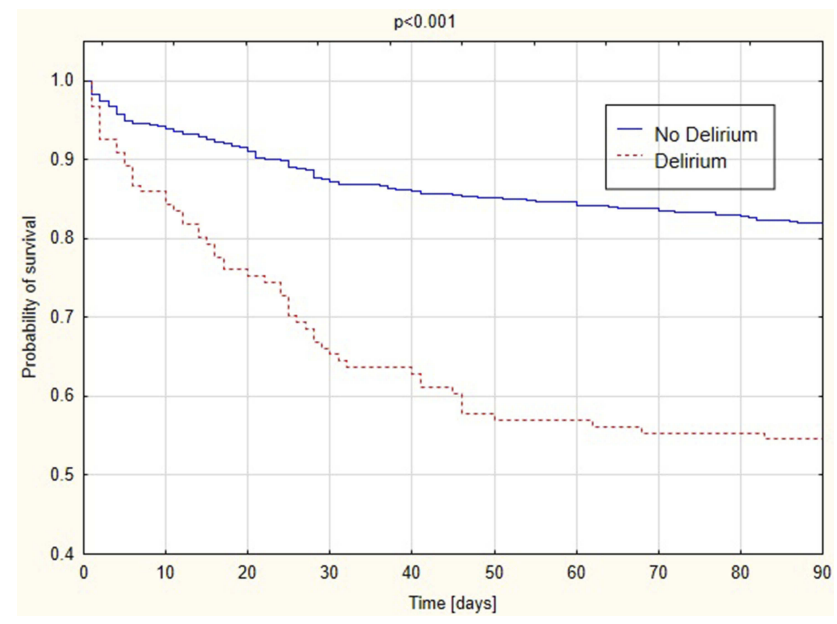

Figure 3 Kaplan-Meier curves - mortality until 90 days in patients with and without delirium. have been regarded as independent risk factors. ${ }^{21}$ Higher age was found as a risk factor in other studies and may be explained by the changes in morphology of aging brains as immunosenescence or inflammaging. ${ }^{27-29}$ Surprisingly, the effect of age has not been shown in our study, probably due to the fact that majority of patients were above 70 years of age.

According to Ahn et al troponin elevation in acute ischemic stroke has high prognostic significance for long-term mortality (risk of death $46 \%$ for patients with troponin elevation vs $17 \%$ for patients without troponin elevation), even after controlling for multiple c-factors contributing to mortality. ${ }^{30}$ The results of our study do not support this observation. The troponin level was indeed elevated in patients with delirium, yet it did not reach significance in multivariable analysis $(\mathrm{p}=0.117)$.

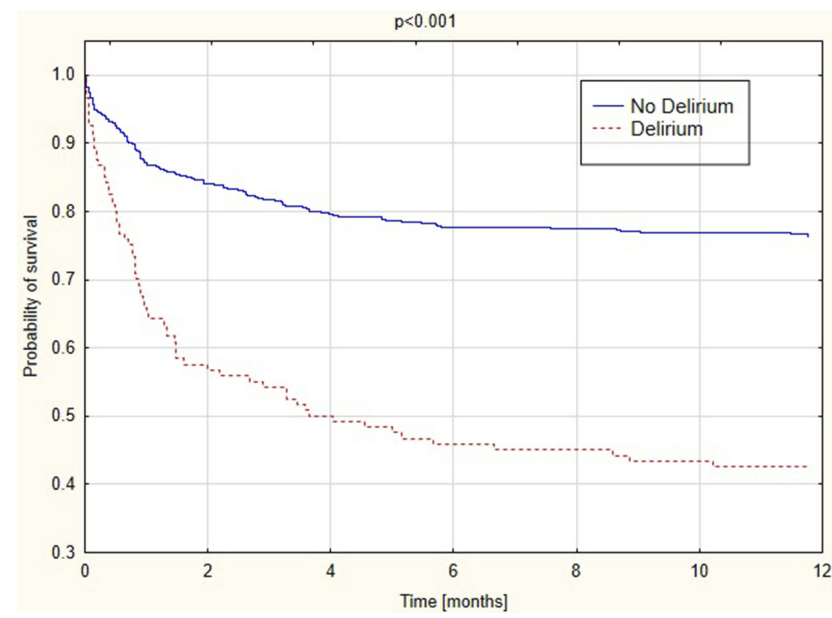

Figure 4 Kaplan-Meier curves - mortality until year I in patients with and without delirium. 
Studies have reported that smoking increases stroke risk and that abrupt smoking cessation may be associated with delirium. Yet other data showed that nicotine use can be a beneficial prognostic factor after AIS, supporting its paradoxical benefit. Lim et al reported that cigarette smoking is an independent risk factor for post-stroke delirium. ${ }^{31}$ The results of our study showed no difference in nicotine use between patients with and without delirium and smoking was not a risk factor for post-stroke delirium. In a casecontrol study performed by Nydahl et al, analysis matched for dementia, severe neurological disability on admission, increased age $\geq 72$ year., and increased CRP on admission showed that delirious patients had more complications but not a worse outcome except a tendency for a reduced rehabilitation improvement, compared to control patients without delirium. $^{32}$

Both previous studies and our results have shown that severe disability on admission, usually assessed by high scores on the modified Rankin Scale, also presented a higher risk for delirium. This can be explained by altered neurotransmission that leads to delirium. ${ }^{16}$

Laboratory values reported in this study showed significant differences between patients with and without delirium. ${ }^{33}$ This study also aimed to determine if simple hematology parameters could be used as risk factors for acute ischemic stroke. ${ }^{34,35}$ We found that mean levels of leukocytes were elevated, and levels of platelets were decreased in patients with stroke and delirium, resulting in a decreased platelet-to WBC ratio, that can be regarded as an independent risk factor of early-onset delirium. Therefore, the results of our study add important evidence to the understanding of a causative relationship between delirium and a dysfunction of immune effector cells. ${ }^{36}$ Furthermore, this finding suggests that the pathophysiology of delirium relies on inadequate response of the immune system to acute brain episodes. Previous small studies have reported that an imbalance between circulating immune cells may be associated with worse neurological outcome in high-risk vascular patients. ${ }^{37}$ A small study performed by Egberts et al has shown that an imbalance between neutrophils and lymphocytes leading to an elevated level of NLR were associated with delirium development in elderly geriatric patients. ${ }^{38}$ It has been suggested that in the mechanism of delirium formation it is the right ratio, or a given balance between immune cells (neutrophils and lymphocytes) is of major importance. ${ }^{38}$ Differences in the level of derived inflammatory white blood cell ratios (NLR or PWR) between patients with and without delirium may reflect a degree of subclinical inflammation in delirious patients. ${ }^{39}$

When analyzing the inflammatory pathomechanism of delirium it is obvious that new diagnostic and prognostic inflammatory markers may be reliable to aid delirium diagnosis. Inflammatory markers used in the research setting are usually of no use in clinical practice. Moreover, available research suggests that the markers and pathways involved in the pathophysiology of delirium may differ between specific groups of patients. ${ }^{40}$ Therefore, simple laboratory factors - CRP, NLR, PWR are of special value to aid the diagnostic process, but their use (including cutoff values) should be designed for certain patient populations. Values derived from blood morphology, platelet count and leucocyte count, namely the platelet-to-WBC ratio may be regarded as a cheap, readily available biomarker, yet further studies are necessary to confirm that the PWR below the level of 20.22 may be used in prognosing post-stroke delirium. The differential white blood cells count analysis is usually done as part of routine blood testing done at admission to the hospital in every patient and can be performed with every automated hematology analyzer.

\section{Study Strengths And Limitations}

Our study has strengths and limitations that deserve a comment. The main strength of this investigation is a very homogenous group of patients including only those with first-ever stroke, large number of patients and the prospective character of the study. Diagnosis of delirium may impose a challenge, especially in neurological patients, because of stroke-related speech or visual disorders, mood fluctuations and cognitive impairment. To overcome these problems and minimize the interobserver bias we used CAM-ICU test for delirium screening that has high sensitivity and specificity and can be used both for verbal and non-verbal patients. ${ }^{10,12}$ Another strength is the selected timeframe for delirium screening previous studies reported that majority of patients develop delirium during the first $24 \mathrm{hrs}^{21}$ Moreover, the authors of a meta-analysis, Shi et al, called for prospective observational studies. ${ }^{1}$

On the other hand, our study is not without limitations. First, this was a single center sample of patients with acute ischemic stroke and the results may not be adequate to other populations of patients with delirium and lacks generalizability. Second, the homogeneity of the study sample (firstever stroke) causes that the results may not be applicable to a larger group of patients (stroke in general). Despite these 
limitations, our study is the first report concentrating on early-onset delirium in first-ever stroke sample of patients. It is advisable to perform further prospective multicenter studies in this groups of patients in different settings to evaluate the role of our preliminary conclusions.

\section{Conclusion}

Nearly $16 \%$ of patients with first ever acute ischemic stroke developed delirium within $24 \mathrm{hrs}$ of admission after the onset of symptoms. More than twice as many patients with delirium developed any kind of complications, the most common being pulmonary, cardiac and renal problems. The probability of 3-months and oneyear survival in patients with delirium was significantly decreased. Atrial fibrillation, higher Rankin score, hemianopia and lower PWR were independently associated with early onset delirium in patients with first ever acute ischemic stroke. This confirms that deprivation of senses and early generalized inflammatory response are critical for delirium development.

\section{Author Contributions}

All authors contributed to data analysis, drafting or revising the article, gave final approval of the version to be published, and agree to be accountable for all aspects of the work.

\section{Data Sharing Statement}

The information regarding deidentified participant data is accessible from the data manager (email: aleksandra. szylinska@gmail.com) only upon a reasonable written request (as per local policy). Patient data will be stored locally for a period of time required by country law and local regulations.

\section{Disclosure}

The authors report no conflicts of interest in this work.

\section{References}

1. Shi Q, Presutti R, Selchen D, Saposnik G. Delirium in acute stroke: a systematic review and meta-analysis. Stroke. 2012;43(3):645-649. doi:10.1161/STROKEAHA.111.643726

2. Jeon SB, Koh Y, Choi HA, Lee K. Critical care for patients with massive ischemic stroke. J Stroke. 2014;16(3):146-160. doi:10.5853/ jos.2014.16.3.146. Epub 2014 Sep 30.

3. Inouye SK, Westendorp RG, Saczynski JS. Delirium in elderly people. Lancet. 2014;383(9920):911-922. doi:10.1016/S0140-6736(13)60688-1

4. McManus J, Pathansali R, Stewart R, Macdonald A, Jackson S. Delirium post-stroke. Age Ageing. 2007;36(6):613-618. doi:10.1093/ ageing/afm140
5. American Psychiatric Association. Diagnostic and Statistical Manual of Mental Disorders: DSM-5. 5th ed. Arlington (VA): American Psychiatric Association; 2013.

6. Girard TD, Thompson JL, Pandharipande PP, et al. Clinical phenotypes of delirium during critical illness and severity of subsequent long-term cognitive impairment: a prospective cohort study. Lancet Respir Med. 2018;6(3):213-222. doi:10.1016/S2213-2600(18)30062-6

7. Kotfis K, Szylińska A, Listewnik M, et al. Early delirium after cardiac surgery: an analysis of incidence and risk factors in elderly ( $\geq 65$ years) and very elderly ( $\geq 80$ years) patients. Clin Interv Aging. 2018;13:1061-1070. doi:10.2147/CIA.S166909

8. Khan BA, Zawahiri M, Campbell NL, Boustani MA. Biomarkers for delirium - a review. $J$ Am Geriatr Soc. 2011;59(0 2):S256-S261. doi:10.1111/j.1532-5415.2011.03702.x

9. Kotfis K, Szylińska A, Listewnik M, Brykczyński M, Ely EW, Rotter I. Diabetes and elevated preoperative $\mathrm{HbAlc}$ level as risk factors for postoperative delirium after cardiac surgery: an observational cohort study. Neuropsychiatr Dis Treat. 2019;15:511-521. doi:10.2147/ NDT.S196973

10. Marra A, Kotfis K, Hosie A, et al. Delirium monitoring: yes or no? That is the question. Am J Crit Care. 2019;28(2):127-135. doi:10.4037/ajec2019874

11. Barr J, Fraser GL, Puntillo K, et al. Clinical practice guidelines for the management of pain, agitation, and delirium in adult patients in the intensive care unit. Crit Care Med. 2013;41(1):263-306. doi:10.1097/CCM.0b013e3182783b72

12. Kotfis K, Zegan-Barańska M, Żukowski M, Kusza K, Kaczmarczyk M, Ely EW. Multicenter assessment of sedation and delirium practices in the intensive care units in Poland - is this common practice in Eastern Europe? BMC Anesthesiol. 2017;17(1):120. doi:10.1186/ s12871-017-0415-2

13. Kotfis K, Marra A, Ely EW. ICU delirium - a diagnostic and therapeutic challenge in the intensive care unit. Anaesthesiol Intensive Ther. 2018;50(2):160-167. doi:10.5603/AIT.a2018.0011

14. Mitasova A, Kostalova M, Bednarik J, et al. Poststroke delirium incidence and outcomes: validation of the Confusion Assessment Method for the Intensive Care Unit (CAM-ICU). Crit Care Med. 2012;40(2):484-490. doi:10.1097/CCM.0b013e318232da12

15. Patel MB, Bednarik J, Lee P, et al. Delirium monitoring in neurocritically ill patients: a systematic review. Crit Care Med. 2018;46 (11):1832-1841. doi:10.1097/CCM.0000000000003349

16. Maldonado JR. Neuropathogenesis of delirium: review of current etiologic theories and common pathways. Am J Geriatr Psychiatry. 2013;21(12):1190-1222. doi:10.1016/j.jagp.2013.09.005

17. van Gool WA, van de Beek D, Eikelenboom P. Systemic infection and delirium: when cytokines and acetylcholine collide. Lancet. 2010;375(9716):773-775. doi:10.1016/S0140-6736(09)61158-2

18. Zaal IJ, Slooter AJ. Delirium in critically ill patients: epidemiology, pathophysiology, diagnosis and management. Drugs. 2012;72:14571471. doi:10.2165/11635520-000000000-00000

19. Egberts A, Wijnbeld EH, Fekkes D, et al. Neopterin: a potential biomarker for delirium in elderly patients. Dement Geriatr Cogn Disord. 2015;39(1-2):116-124. doi:10.1159/000366410

20. MacLullich AM, Edelshain BT, Hall RJ, et al. Cerebrospinal fluid interleukin-8 levels are higher in people with hip fracture with perioperative delirium than in controls. J Am Geriatr Soc. 2011;59 (6):1151-1153. doi:10.1111/j.1532-5415.2011.03428.x

21. Qu J, Chen Y, Luo G, Zhong H, Xiao W, Yin H. Delirium in the acute phase of ischemic stroke: incidence, risk factors, and effects on functional outcome. J Stroke Cerebrovasc Dis. 2018;27(10):26412647. doi:10.1016/j.jstrokecerebrovasdis.2018.05.034

22. Pasinska P, Kowalska K, Klimiec E, Szyper-Maciejowska A, Wilk A, Klimkowicz-Mrowiec A. Frequency and predictors of post-stroke delirium in PRospective Observational POLIsh Study (PROPOLIS). $J$ Neurol. 2018;265(4):863-870. doi:10.1007/s00415-018-8782-2 
23. Pasinska P, Kowalska K, Wilk A, Szyper-Maciejowska A, Klimkowicz-Mrowiec A. Post-stroke delirium course in Prospective Observational Polish Study (PROPOLIS). Neuropsychiatry (London). 2019;9(2):2279-2285.

24. Gustafson Y, Eriksson O, Sture T, Bucht A, Go“sta K. Acute confusional states (delirium) in stroke patients. Cerebrovasc Dis. 1991;1:257-264. doi:10.1159/000108852

25. Dostovic Z, Smajlovic D, Sinanovic O, Vidovic M. Duration of delirium in the acute stage of stroke. Acta Clin Croat. 2009;48:13-17.

26. Caeiro L, Ferro JM, Albuquerque R, Figueira ML. Delirium in the first days of acute stroke. J Neurol. 2004;251:171-178. doi:10.1007/ s00415-004-0294-6

27. Carin-Levy G, Mead GE, Nicol K, Rush R, van Wijck F. Delirium in acute stroke: screening tools, incidence rates and predictors: a systematic review. J Neurol. 2012;259(8):1590-1599. doi:10.1007/s00415-011-6383-4

28. Oldenbeuving AW, de Kort PL, Jansen BP, et al. Delirium in the acute phase after stroke: incidence, risk factors, and outcome. Neurology. 2011;76:993-999. doi:10.1212/WNL.0b013e318210411f

29. Miu DK, Yeung JC. Incidence of post-stroke delirium and 1-year outcome. Geriatr Gerontol Int. 2013;13:123-129. doi:10.1111/ j.1447-0594.2012.00871.x

30. Ahn SH, Lee JS, Kim YH, et al. Prognostic significance of troponin elevation for long-term mortality after ischemic stroke. J Stroke. 2017;19(3):312-322. doi:10.5853/jos.2016.01942. Epub 2017 Sep 6.

31. Lim TS, Lee JS, Yoon JH, et al. Cigarette smoking is an independent risk factor for post-stroke delirium. BMC Neurol. 2017;17(1):56. doi:10.1186/s12883-017-0840-3

32. Nydahl P, Bartoszek G, Binder A, et al. Prevalence for delirium in stroke patients: a prospective controlled study. Brain Behav. 2017;7 (8):e00748. doi:10.1002/brb3.748
33. Simone MJ, Tan ZS. The role of inflammation in the pathogenesis of delirium and dementia in older adults: a review. CNS Neurosci Ther. 2011;17:506-513. doi:10.1111/j.1755-5949.2010.00173.x

34. Dhabhar FS, Malarkey WB, Neri E, McEwen BS. Stress-induced redistribution of immune cells - from barracks to boulevards to battlefields: a tale of three hormones - Curt Richter Award winner. Psychoneuroendocrinology. 2012;37(9):1345-1368. doi:10.1016/j. psyneuen.2012.05.008

35. Kolaczkowska E, Kubes P. Neutrophil recruitment and function in health and inflammation. Nat Rev Immunol. 2013;13(3):159-175. doi: $10.1038 /$ nri3399

36. Chung HY, Kim DH, Lee EK, et al. Redefining chronic inflammation in aging and age-related diseases: proposal of the senoinflammation concept. Aging Dis. 2019;10(2):367-382. doi:10.14336/ AD.2018.0324

37. Kotfis K, Biernawska J, Zegan-Barańska M, Żukowski M. Peripheral blood lymphocyte subsets (CD4+, CD8+ T cells, NK cells) in patients with cardiovascular and neurological complications after carotid endarterectomy. Int J Mol Sci. 2015;16(5):10077-10094. doi:10.3390/ijms 160510077

38. Egberts A, Mattace-Raso FUS. Increased neutrophil-lymphocyte ratio in delirium: a pilot study. Clin Interv Aging. 2017;12:1115-1121. doi:10.2147/CIA.S137182

39. Zahorec R. Ratio of neutrophil to lymphocyte counts-rapid and simple parameter of systemic inflammation and stress in critically ill. Bratisl Lek Listy. 2001;102(1):5-14.

40. Egberts A, Osse RJ, Fekkes D, Tulen JHM, van der Cammen TJM, Mattace-Raso FUS. Differences in potential biomarkers of delirium between acutely ill medical and elective cardiac surgery patients. Clin Interv Aging. 2019;14:271-281. doi:10.2147/CIA.S193605
Clinical Interventions in Aging

\section{Publish your work in this journal}

Clinical Interventions in Aging is an international, peer-reviewed journal focusing on evidence-based reports on the value or lack thereof of treatments intended to prevent or delay the onset of maladaptive correlates of aging in human beings. This journal is indexed on PubMed Central, MedLine, CAS, Scopus and the Elsevier
Bibliographic databases. The manuscript management system is completely online and includes a very quick and fair peer-review system, which is all easy to use. Visit http://www.dovepress.com/ testimonials.php to read real quotes from published authors. 